\title{
Effect of Time Elapsed since Gadolinium Administration on Atherosclerotic Plaque Enhancement in Clinical Vessel Wall MR Imaging Studies
}

\author{
(D) A. de Havenon, (DH.J. Muhina, (DD.L. Parker, (D).S. McNally, and (D).D. Alexander
}

\begin{abstract}
SUMMARY: Vessel wall MR imaging is a useful tool for the evaluation of intracranial atherosclerotic disease. Enhancement can be particularly instructive. This study investigated the impact of the duration between contrast administration and image acquisition. The cohort with the longest duration had the greatest increase in signal intensity change. When using vessel wall MR imaging to assess intracranial atherosclerotic disease, protocols should be designed to maximize the duration between contrast administration and image acquisition to best demonstrate enhancement.
\end{abstract}

ABBREVIATIONS: DANTE = delay alternating with nutation for tailored excitation; vwMRI = vessel wall MR imaging; ICAD = intracranial atherosclerotic disease; SPACE = sampling perfection with application-optimized contrasts by using different flip angle evolutions

D evelopment of vessel wall MR imaging (vwMRI) protocols has improved the evaluation of intracranial atherosclerotic disease (ICAD) by providing direct visualization of the vessel wall and the plaque itself. ${ }^{1-6}$ In the evaluation of ICAD with vwMRI, an important diagnostic finding is plaque enhancement, a characteristic widely believed to reflect inflammation. ${ }^{7,8}$ Inconsistencies in acquisition parameters and image interpretation have limited progress in the development of these promising MR imaging techniques. ${ }^{6,79-12}$

Reproducible quantitative interpretation techniques could help overcome these limitations, particularly with respect to enhancement, but standardization of acquisition parameters is also needed. ${ }^{6}$ Duration between contrast administration and image acquisition affects the degree of measured enhancement in other pathologies. $^{13,14}$ This study examined the impact of time intervals on enhancement measured in ICAD plaques and reference structures.

\section{MATERIALS AND METHODS}

Following an institutional review board-approved protocol, retrospective analysis was performed of patients undergoing vwMRI

Received May 20, 2019; accepted after revision July 17.

From the Departments of Radiology and Imaging Sciences (M.D.A., D.L.P. J.S.M.), Neurosurgery (M.D.A.), Neurology (A.d.H.), and the School of Medicine (H.J.M.), University of Utah, Salt Lake City, Utah.

This study was funded, in part, by National Institutes of Health grants NIH S10OD018482, NIH R01HL127582, NIH/NINDS K23NS105924, and the American Heart Association (19TPA34910194 and 17SDG33460420).

Please address correspondence to Matthew D. Alexander, MD, 30 North 1900 East, Room 1A071, Salt Lake City, UT 84132; e-mail: Matthew.Alexander@hsc.utah.edu

-- Indicates open access to non-subscribers at www.ajnr.org

http://dx.doi.org/10.3174/ajnr.A6191 for the evaluation of ischemic stroke at a major academic medical center. In this protocol, vwMRI studies are performed for patients with confirmed new infarcts suspected of being due to ICAD or not attributed to another etiology. All patients in this study were evaluated with vwMRI within 14 days of the infarct.

Studies were performed with dedicated head coils on Prisma, Trio, or Verio 3T MR imaging scanners (Siemens, Erlangen, Germany). Two blinded neuroradiologists assessed the arterial tree upstream from the new infarct. The reviewers were notified about which artery to assess by the vascular neurologist, who adjudicated the stroke parent artery status according to diffusion-weighted imaging lesions within the vascular territory of a major artery with ICAD. Reviewers were blinded to clinical data and other MR imaging findings, most notably DWI. Reviewers noted the lesion that had most likely caused the downstream infarction. The culprit lesions were determined by each reviewer and confirmed between them to be the same lesion for each patient. Each reviewer was blinded to measurements made by the other reviewer. Patients in whom multiple ICAD lesions in the same vascular bed could be considered culprit were excluded to avoid bias.

After we confirmed the consensus on the culprit lesion, maximum signal intensity values were recorded on 3D T1-weighted sampling perfection with application-optimized contrasts by using different flip angle evolutions (SPACE; Siemens) with delay alternating with nutation for tailored excitation (DANTE) flow suppression pre- and postcontrast. ${ }^{5}$ Patients were given gadobenate dimeglumine (MultiHance; Bracco Diagnostics, Princeton, New Jersey) at a dose of $0.2 \mathrm{~mL} / \mathrm{kg}(0.1 \mathrm{mmol} / \mathrm{kg})$. Additionally, structures known to normally enhance were included, including the low infundibulum, defined as the lowest segment distinguishable from the pituitary gland on axial images; muscle, chosen 


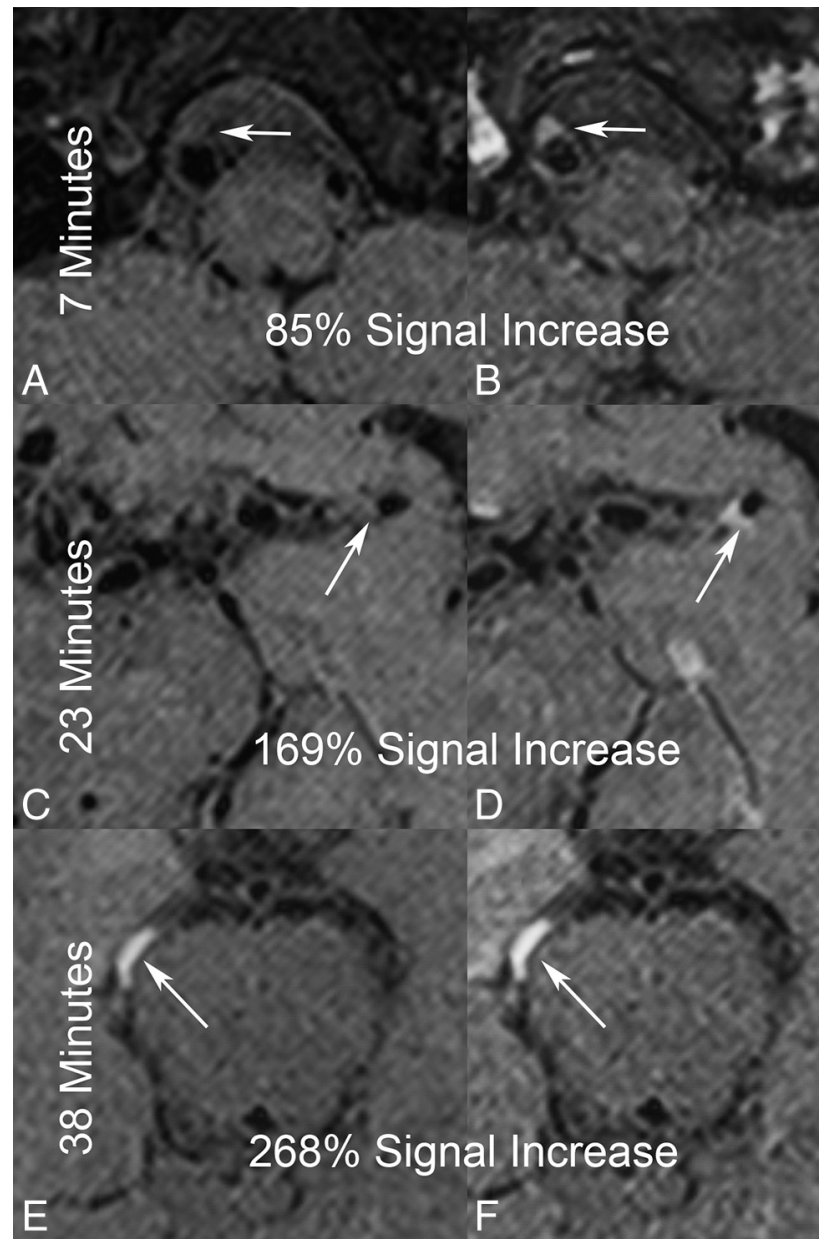

FIG 1. Representative images of VwMRI studies of symptomatic ICAD lesions (arrows) in each of the 3 timing cohorts, $<20$ minutes ( $A$ and $B$ ), 20-30 minutes ( $C$ and $D$ ), and 30-40 minutes ( $E$ and $F$ ). For each study, pre- $(A, C$, and $E)$ and postcontrast $(B, D, F)$ images are shown of lesions in the right $\mathrm{V} 4$ segment $(A$ and $B)$, distal left Ml segment ( $E$ and $F)$.

within the temporalis muscle medial to its midbelly fibrous band; cavernous sinus, measuring a segment clearly representing only blood; and the choroid plexus. ${ }^{6,15}$ Three data points were measured for each site assessed, and the mean was calculated.

Times were tabulated for contrast administration and acquisition of postcontrast images. To exclude outlier data during early development of the vwMRI protocol that could introduce bias, we excluded studies with $>40$ minutes between contrast injection and $\mathrm{T} 1$ DANTE acquisition. Correlation coefficients were calculated among variables as well as $P$ values to assess the significance of associations. For further analysis, the cohort was divided into 3 different time intervals from contrast injection to T1 DANTE: 0-20 minutes, 2030 minutes, and 30-40 minutes. Comparison was made of the variance of pre- to postcontrast measurements across these time periods using the Levene test of the equality of variance. All analyses were performed in STATA 15.1 (StataCorp, College Station, Texas).

\section{RESULTS}

Studies from 54 patients were evaluated. Thirty-five patients met all the inclusion criteria (10 studies in the 0- to 20-minute group,

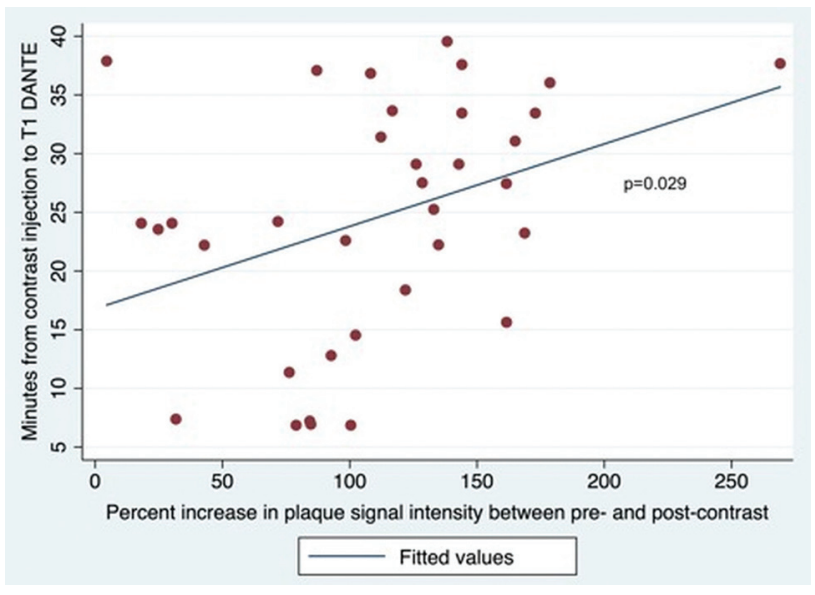

FIG 2. Linear regression between the minutes from contrast injection to TI DANTE and the percentage increase in plaque signal intensity between pre- and postcontrast.

13 at $20-30$ minutes, and 12 at $30-40$ minutes). Representative studies from each time group are provided in Fig 1. The mean \pm SD time from contrast injection to T1 DANTE was $24.5 \pm$ 10.4 minutes (range, 6.9-39.5 minutes). In culprit plaques, the percentage increase in signal intensity from pre- to postcontrast was $110.2 \% \pm 54.7 \%$, which was significantly associated with the time from contrast injection to T1 DANTE (Fig 2, $P=.029$ ). For the reference structures, there was no association between the time from contrast injection to T1 DANTE and the change in signal intensity between pre- and postcontrast (Table). Creating a ratio of percentages in increased $\mathrm{T} 1$ signals of plaque over the lower infundibulum demonstrates a stronger correlation with timing than plaque alone $\left(r^{2}=0.410, P=.016\right)$. If one introduces a pituitary signal increase into the model for plaque as a covariate, plaque maintains the association with time $(P<.05)$. Additionally, there is no direct association between the plaque and infundibulum changes $\left(r^{2}=-0.103, P=.561\right)$.

\section{DISCUSSION}

Evaluation of ICAD can be aided by vwMRI studies. ${ }^{1-3,6,8}$ While these techniques have proved useful, broad clinical use is impeded by heterogeneous acquisition and interpretation methods. Standardized methodologies can mitigate such issues, particularly when quantitative analyses are used. ${ }^{6}$ Enhancement of ICAD plaques is a particularly instructive feature, and this characteristic, in particular, is prone to variability. ${ }^{6-8}$ These results confirm our early clinical observation that lesion enhancement was accentuated by increased duration between contrast administration and acquisition of postcontrast DANTE images. The association between time and T1 signal changes is independent of changes in the pituitary gland. Standardizing plaque signal change to change measured in the pituitary gland is even more dependent on time from contrast administration. In response to these findings, we have standardized a vwMRI protocol that maximizes this duration within the accepted temporal confines for these studies by acquiring sequences not impacted by contrast (T2, FLAIR) after contrast administration and before postcontrast DANTE imaging. 
Correlation between time from contrast injection to T1 DANTE and measurements of change in signal intensity between pre- and postcontrast

\begin{tabular}{|c|c|c|c|}
\hline Variable & Correlation Coefficient & Coefficient of Determination $\left(r^{2}\right)$ & $P$ Value \\
\hline \multicolumn{4}{|l|}{ Time from contrast injection to TI DANTE } \\
\hline Plaque (\% increase) & 0.370 & 0.137 & .029 \\
\hline Low infundibulum (\% increase) $(n=34)$ & -0.314 & 0.099 & .071 \\
\hline Cavernous sinus (\% increase) $(n=32)$ & -0.054 & 0.003 & .770 \\
\hline Muscle $(\%$ increase $)(n=33)$ & -0.011 & $<0.001$ & .951 \\
\hline Choroid plexus (\% increase) $(n=33)$ & 0.049 & 0.002 & .788 \\
\hline \multicolumn{4}{|c|}{ Change in signal intensity between pre- and postcontrast } \\
\hline Plaque (\% increase) & 0.370 & 0.137 & .029 \\
\hline Low infundibulum (\% increase) $(n=34)$ & -0.314 & 0.099 & .071 \\
\hline Cavernous sinus (\% increase) $(n=32)$ & -0.054 & 0.003 & .770 \\
\hline Muscle (\% increase) $(n=33)$ & -0.011 & $<0.001$ & .951 \\
\hline Choroid plexus (\% increase) $(n=33)$ & 0.049 & 0.002 & .788 \\
\hline
\end{tabular}

This study has several limitations that warrant mention. Visualized enhancement may reflect variables other than timing such as differences in plaques, patient age or sex, or other factors. Additionally, elapsed time after contrast may be confounded, which cannot be determined without imaging the same person multiple times or doing a dynamic study. Such factors can be assessed in future investigations. Despite these limitations, it appears that future studies using vwMRI might benefit from defined time intervals between contrast administration and postcontrast T1 imaging.

Disclosures: J. Scott McNally—RELATED: Grant: American Heart Association, Comments: American Heart Association Scientist Development Grant (17SDG33460420) supports a portion of my research time.* *Money paid to the institution.

\section{REFERENCES}

1. Mossa-Basha M, Alexander M, Gaddikeri S, et al. Vessel wall imaging for intracranial vascular disease evaluation. J Neurointerv Surg 2016;8:1154-59 CrossRef Medline

2. de Havenon A, Mossa-Basha M, Shah L, et al. High-resolution vessel wall MRI for the evaluation of intracranial atherosclerotic disease. Neuroradiology 2017;59:1193-1202 CrossRef Medline

3. Mandell DM, Mossa-Basha M, Qiao Y, et al. Intracranial vessel wall MRI: principles and expert consensus recommendations of the American Society of Neuroradiology. AJNR Am J Neuroradiol 2017;38:218-29 CrossRef Medline

4. Li L, Chai JT, Biasiolli L, et al. Black-blood multicontrast imaging of carotid arteries with DANTE-prepared 2D and 3D MR imaging. Radiology 2014;273:560-69 CrossRef Medline

5. Li L, Miller KL, Jezzard P. DANTE-prepared pulse trains: a novel approach to motion-sensitized and motion-suppressed quantitative magnetic resonance imaging. Magn Reson Med 2012;68:142338 CrossRef Medline
6. Alexander MD, de Havenon A, Kim SE, et al. Assessment of quantitative methods for enhancement measurement on vessel wall magnetic resonance imaging evaluation of intracranial atherosclerosis. Neuroradiology 2019;61:643 CrossRef

7. Gupta A, Baradaran H, Al-Dasuqi K, et al. Gadolinium enhancement in intracranial atherosclerotic plaque and ischemic stroke: a systematic review and meta-analysis. J Am Heart Assoc 2016;5 CrossRef

8. Alexander MD, Yuan C, Rutman A, et al. High-resolution intracranial vessel wall imaging: imaging beyond the lumen. J Neurol Neurosurg Psychiatry 2016;87:589-97 CrossRef Medline

9. Kim JM, Jung $\mathrm{KH}$, Sohn $\mathrm{CH}$, et al. Middle cerebral artery plaque and prediction of the infarction pattern. Arch Neurol 2012;69:147075 CrossRef Medline

10. Qiao Y, Zeiler SR, Mirbagheri S, et al. Intracranial plaque enhancement in patients with cerebrovascular events on high-spatial-resolution MR images. Radiology 2014;271:534-42 CrossRef Medline

11. Skarpathiotakis $\mathrm{M}$, Mandell $\mathrm{DM}$, Swartz $\mathrm{RH}$, et al. Intracranial atherosclerotic plaque enhancement in patients with ischemic stroke. AJNR Am J Neuroradiol 2013;34:299-304 CrossRef Medline

12. Vakil P, Vranic J, Hurley MC, et al. T1 gadolinium enhancement of intracranial atherosclerotic plaques associated with symptomatic ischemic presentations. AJNR Am J Neuroradiol 2013;34:2252-58 CrossRef Medline

13. Tofts PS, Kermode AG. Measurement of the blood-brain barrier permeability and leakage space using dynamic MR imaging, 1: fundamental concepts. Magn Reson Med 1991;17:357-67 CrossRef Medline

14. Alexander MD, Hughes N, Cooke DL, et al. Revisiting classic MRI findings of venous malformations: changes in protocols may lead to potential misdiagnosis. Neuroradiol J 2018;31:5095-12 CrossRef Medline

15. Smirniotopoulos JG, Murphy FM, Rushing EJ, et al. Patterns of contrast enhancement in the brain and meninges. Radiographics 2007;27:525-51 CrossRef Medline 